\title{
Clinical analysis of temporary pacemaker implantation in 13 children
}

\author{
Ting-Ting Li, Ji Cheng \\ Department of Cardiology, Tianjin Children's Hospital/Tianjin University Children's Hospital, Tianjin, China \\ Contributions: (I) Conception and design: TT Li; (II) Administrative support: J Cheng; (III) Provision of study materials or patients: TT Li; (IV) \\ Collection and assembly of data: TT Li; (V) Data analysis and interpretation: TT Li; (VI) Manuscript writing: Both authors; (VII) Final approval of \\ manuscript: Both authors. \\ Correspondence to: Ting-Ting Li. Department of Cardiology, Tianjin Children's Hospital/Tianjin University Children's Hospital, 225 Machang Road, \\ Tianjin 300074, China. Email: litingting201706@163.com.
}

Background: At present, temporary pacemaker implantation is very common in the treatment of cardiovascular diseases in adults. However, the number of pediatric pacemakers implanted is still relatively small, and relevant research is also far less than that of adults. This study aimed to explore the application of temporary pacemakers in children with acute and critical cardiovascular diseases.

Methods: The clinical data of children with cardiovascular diseases who were treated with temporary pacemakers in Tianjin Children's Hospital from October 2017 to February 2021 were analyzed retrospectively.

Results: A total of 13 children with cardiovascular diseases were included in this study, including 4 males and 9 females, mean age of $71.2 \pm 56.3$ months, and median body weight of $15.5 \mathrm{~kg}$. There were 9 children with endocardial pacing and 4 children with epicardial pacing. The types of diseases included fulminant myocarditis $(n=8)$, complete atrioventricular block (CAVB; $n=1)$, and arrhythmias after open heart surgery $(\mathrm{n}=4)$. The median time from onset to admission was 1.0 days in children with endocardial pacing and there was cardiac arrest in 2 children, heart failure in 9 children, cardiogenic shock in 8 children, and AdamsStokes attack in 7 children. The median time from admission to implantation of temporary pacemakers was $3.0 \mathrm{~h}$ and the operation time was $55.0 \pm 19.4 \mathrm{~min}$. All 4 children with epicardial pacing had pacemakers implanted during operation because of CAVB. The pacing mode was VVI mode. The initial perceptual voltage was 1-2 mv, the output voltage was $5 \mathrm{v}$, and the pacing frequency was $70-145 \mathrm{bpm}$. A total of 11 children reverted to sinus rhythm within $5.0(1.8-34.0) \mathrm{h}$ and the working time of temporary pacemakers was 134.0 (15.0-191.0) h. There was poor pacing in 2 children and catheter displacement in 1 child during pacing. A total of 12 children were followed up for $20.0 \pm 12.5$ months and 1 was lost to follow-up. During the follow-up period, the cardiac functions were basically normal and no new arrhythmia appeared.

Conclusions: Temporary pacemakers have the advantage of simple operation, definite effect, and safety which has a remarkable effect in the treatment of acute and critical cardiovascular diseases in children.

Keywords: Temporary pacemaker; children; cardiovascular disease; acute and critical; arrhythmia

Submitted Sep 26, 2021. Accepted for publication Jan 30, 2022.

doi: $10.21037 / \mathrm{tp}-21-586$

View this article at: https://dx.doi.org/10.21037/tp-21-586 


\section{Introduction}

A pacemaker Isan adjustable artificial electrical pulse generator, the use of which, through effective depolarization and cardiac contraction, appropriate heart rate and cardiac output can be obtained to treat cardiac dysfunction $(1,2)$. A temporary pacemaker is an electronic device with nonpermanent implantation of a pacing lead (1). At present, the implantation of a temporary pacemaker is very common in the treatment of cardiovascular diseases in adults, and its technology is mature and effective. In recent years, with the improvement of the reliability of pacing systems, the development of pacing technology, and the increase of clinical experience, temporary pacemakers are increasingly used in the treatment of acute and critical cardiovascular diseases in children, such as fulminant myocarditis, malignant arrhythmia, and arrhythmia after open heart surgery. When there is symptomatic or worrying bradycardia or tachycardia that ineffective to drug therapy or requiring repeated cardioversion, temporary pacemakers are needed. As an effective means of transition and protection, temporary pacemakers are used to improve success rate of rescue and improve prognosis (3-10). However, some difficulties are involved in the temporary pacemaker implantation in children, such as small physique, cardiac anomaly, lower tolerance to disease, many complications and so on and the number of pediatric pacemakers implanted is still relatively small. Pediatric pacemaker implants comprise $<1 \%$ of all pacemaker implants $(11,12)$. The relevant research is also far less than that of adults. In the previous reports of temporary pacemaker implantation in children, most of them are case reports or studies on the application of temporary pacemakers for a certain disease, but there was little comprehensive analysis on the application of temporary pacemakers in children with acute and critical cardiovascular diseases. In this study, the clinical data of children with cardiovascular diseases who were treated with temporary pacemakers in Tianjin Children's Hospital from October 2017 to February 2021 were retrospectively analyzed to explore the application of temporary pacemakers in children with acute and critical cardiovascular diseases. We present the following article in accordance with the STROBE reporting checklist (available at https://tp.amegroups.com/ article/view/10.21037/tp-21-586/rc).

\section{Methods}

Children who were treated with temporary pacemakers in
Tianjin Children's Hospital from October 2017 to February 2021 were retrospectively included in this study through review of the electronic medical record system. The case retrieval codes included 37.8001, 37.7800, and 39.6400. All procedures performed in this study involving human participants were in accordance with the Declaration of Helsinki (as revised in 2013). The study was approved by Ethics and Research Committee of Tianjin Children's Hospital (No. L2021-18). Individual consent for this retrospective analysis was waived. The collected data included demographic characteristics, disease types, clinical symptoms, implantation time of temporary pacemakers, pacing mode, pacing parameters, clinical outcomes after implantation [changes of ultrasound cardiogram (UCG), electrocardiogram (ECG) and myocardial enzyme], working time, and follow-ups.

\section{Statistical analysis}

The software SPSS 16.0 (IBM Corporation, Armonk, NY, USA) was used for the statistical analysis. Continuous variables with normal distribution were expressed using mean \pm standard deviations. Continuous variables with non-normal distribution were expressed using median (interquartile range) and the comparison between the groups was detected using Mann-Whitney $\mathrm{U}$ test in nonparametric testing. A $\mathrm{P}$ value of less than 0.05 was considered statistically significant.

\section{Results}

\section{Demographic characteristics}

A total of 13 children with cardiovascular diseases were included in this study, including 4 males and 9 females, aged 2-154 months (mean $71.2 \pm 56.3$ months), body weight $4.5-54.5 \mathrm{~kg}$ (median body weight $15.5 \mathrm{~kg}$ ), and body length $54-155 \mathrm{~cm}$ (mean $106.5 \pm 35.5 \mathrm{~cm}$ ). There were 9 children ( 3 males and 6 females) with endocardial pacing, aged 32-154 months (mean 99.3 \pm 42.7 months), body weight $14.3-54.5 \mathrm{~kg}$ (median body weight $24.0 \mathrm{~kg}$ ) and 4 children ( 1 male and 3 females) with epicardial pacing, aged 2-18 months, body weight $4.5-7.4 \mathrm{~kg}$.

\section{Clinical data before implantation of temporary pacemaker}

All 13 children underwent routine 12-lead ECG after admission. There was complete atrioventricular block 
(CAVB) in 13 children, 2:1 atrioventricular block (2:1 AVB) in 1 child, R-on- $T$ extra systole in 1 child, torsades de pointes ventricular tachycardia $(\mathrm{TdP})$ in 1 child, prolonged QT interval in 1 child, ventricular tachycardia in 4 children, ventricular premature beats in 5 children, and low voltage in 1 child. The 9 children with endocardial pacing included 8 children with fulminant myocarditis and 1 with CAVB. The time from onset to admission was $3 \mathrm{~h}$ to 7 days and the median time was 1.0 days. The clinical symptoms were syncope, convulsion, and disturbance of consciousness in 7 children, abdominal pain and vomiting in 6 children, and chest tightness and fatigue in 4 children. The ventricular rate of admission physical examination was $27-65 \mathrm{bpm}$ (mean $45.9 \pm 12.5 \mathrm{bpm}$ ). There was cardiac arrest in 2 children, heart failure in 9 children, cardiogenic shock in 8 children, and Adams-Stokes attack in 7 children. All 9 children underwent UCG before implantations of temporary pacemakers and 7 children had positive results. The diameter of the heart chamber was increased in 5 children, with 3 cases involving the left atrium, 1 case involving biatrium, and 1 case involving the whole heart. Pericardial effusion occurred in 1 patient. The left ventricular systolic function was decreased in 5 children, left ventricular ejection fraction (LVEF) was $57.1 \% \pm 11.5 \%$, and left ventricular fractional shortening (LVFS) was $30.2 \% \pm 8.6 \%$. Mitral regurgitation occurred in 9 children, mostly small or moderate. Pro-B-type natriuretic peptide (pro-BNP) was increased in all children $(4,443.0 \pm$ 3,853.9 pmol/L). All 4 children with epicardial pacing were implanted during operation, including 3 children with ventricular septal defect repair, and 1 with atrial septal defect plus ventricular septal defect repair, all of whom had CAVB.

\section{Application of temporary pacemakers}

\section{Temporary pacemaker model}

The temporary pacemaker adopted the Reocor S single chamber pacemaker (BIOTRONIK Company, Berlin, Germany). The pacing electrode catheter adopted the ordinary bipolar temporary pacing electrode catheter (St. Jude Medical Company, St Paul, MN, USA).

\section{Implantation of temporary pacemaker Endocardial pacing}

Patients took the supine position, after which general anesthesia and disinfecting of the surgical site were performed, and the right femoral vein pathway was established by Seldinger technique. The catheter was inserted into the right femoral vein and the bipolar temporary pacing catheter was sent to the right ventricular apex under X-ray fluoroscopy. The catheter was maintained at a certain tension in the cardiac cavity and the electrode was close to the myocardium. The intra-cardiac electrogram was measured and the pacing frequency, and perceptual voltage and output voltage were set. The pacing catheter was coiled on the body surface, fixed with a suture, and covered with film.

\section{Epicardial pacing}

The cathodic electrode was attached to the bare area of the blood vessels on the surface of the right ventricle to find the lowest threshold point to fix it. Part of the pacing wire was in the thoracic cavity and the anodic electrode was fixed under the skin near the incision.

\section{Application of temporary pacemakers}

The application of temporary pacemakers in 13 children is shown in Table 1. The time from admission to implantation of temporary pacemakers was $1.5-14.0 \mathrm{~h}$ (median time $3.0 \mathrm{~h}$ ) and the operation time was $55.0 \pm 19.4 \mathrm{~min}$ in 9 children with endocardial pacing. All 4 children with epicardial pacing were implanted with pacemakers during surgery. All temporary pacemakers were implanted successfully for a single time. The electrode arrival rate was $100 \%$ and the right ventricular effective pacing rate was $100 \%$. The pacing mode was VVI mode. The initial perceptual voltage was $1-2 \mathrm{mv}$, the output voltage was $5 \mathrm{v}$, and the pacing frequency was $70-145 \mathrm{bpm}$. After operation, ECG was closely monitored and pacing frequency, perceptual voltage, and output voltage were adjusted according to the changes of ECG. After implantation of temporary pacemakers, 11 children reverted to sinus rhythm within $5.0 \mathrm{~h}(1.8-34.0 \mathrm{~h})$ (7 children with endocardial pacing $22.4 \pm 19.1 \mathrm{~h}$ and 4 children with epicardial pacing $1.5-5.0 \mathrm{~h}$ ). Temporary pacemakers were removed successfully in 11 children ( 7 children with endocardial pacing and 4 children with epicardial pacing). The working time of temporary pacemakers was $134.0 \mathrm{~h}(15.0-191.0 \mathrm{~h})$ (7 children with endocardial pacing $165.1 \pm 32.7 \mathrm{~h}$ and 4 children with epicardial pacing $12.0-17.0 \mathrm{~h}$ ).

There were 2 children with endocardial pacing who did not revert to normal sinus rhythm. One of them was implanted with a permanent pacemaker and the other gave up treatment. Poor pacing occurred in 2 children with endocardial pacing and after increasing the output 
Table 1 Application of temporary pacemaker implantation in 13 children

\begin{tabular}{|c|c|c|c|c|c|c|}
\hline No. & Pacing setting & $\begin{array}{l}\text { Time of implantation } \\
\text { (h) }\end{array}$ & $\begin{array}{l}\text { Pacing frequency } \\
\text { (bpm) }\end{array}$ & $\begin{array}{l}\text { Time of reverting to } \\
\text { sinus rhythm (h) }\end{array}$ & $\begin{array}{l}\text { Time of removal } \\
\text { (h) }\end{array}$ & Outcome \\
\hline 1 & Endocardial pacing & 14.0 & 75 & Not reverted & 100.0 & Not Cured \\
\hline 3 & Endocardial pacing & 2.0 & 70 & 11.0 & 192.0 & Fine \\
\hline 4 & Endocardial pacing & 2.7 & 80 & 0.5 & 134.0 & Fine \\
\hline 6 & Endocardial pacing & 4.5 & 75 & 1.8 & 170.0 & Fine \\
\hline 7 & Endocardial pacing & 3.0 & 80 & 45.0 & 114.0 & Fine \\
\hline 8 & Endocardial pacing & 3.5 & 80 & 34.0 & 154.0 & Fine \\
\hline 11 & Epicardial pacing & During operation & 120 & 1.5 & 12.0 & Fine \\
\hline 12 & Epicardial pacing & During operation & 130 & 3.0 & 14.0 & Fine \\
\hline 13 & Epicardial pacing & During operation & 145 & 2.5 & 15.0 & Fine \\
\hline
\end{tabular}

voltage the pacemakers worked well. Catheter displacement occurred in 1 child with endocardial pacing and after readjusting the position of the pacing catheter and the pacemaker worked well. There were no occurrences of complications such as myocardial perforation, cardiac tamponade, pacemaker-related arrhythmia, catheter rupture, thromboembolism, puncture site infection, difficulty in wires removal, and hemopericardium.

\section{Areas of attention in application of temporary pacemaker in children}

The following procedures were found to assist the process of temporary pacemaker application: (I) prompt pacification of children to avoid complications such as catheter displacement, catheter rupture, puncture site bleeding, and infection; (II) observe ECG monitoring closely and adjust pacemaker parameters to ensure pacing on demand; (III) remove the temporary pacemaker as soon as possible after the condition had stabilized.

\section{Clinical outcomes after implantation of temporary pacemaker}

The 7 children with endocardial pacing who successfully reverted to normal sinus rhythm were all children with fulminant myocarditis. The temporary pacemaker provided effective pacing rate and improved hemodynamics. The positive inotropic drugs were gradually stopped. These 7 children underwent UCG again 3 days after operation. The diameter of the heart chamber returned to normal in 6 and 1 had a slightly larger left atrium. The left ventricular systolic function returned to normal in all 7 children. The LVEF was $67.0 \% \pm 5.7 \%$ and LVFS was $35.3 \% \pm 5.1 \%$. Mitral regurgitation remained in 5 children, mostly small or moderate. There were 5 children with pericardial effusion, mostly light, and only 1 moderate. Thickening of interventricular septum and left ventricular posterior wall occurred in 2 children. The level of myocardial enzyme decreased gradually after operation, and the difference was statistically significant $(\mathrm{P}<0.01)$ (Table 2). At discharge, ECG and ambulatory ECG (Holter) showed that there were 6 children with ventricular premature beats, 1 with R-on-T extrasystole, 3 with ventricular tachycardia, 2 with atrial premature beats, 2 with right bundle branch block, 1 with left bundle branch block, and 1 with $T$ wave change. At discharge, UCG showed that all 7 children with normal heart chamber diameter $(100.0 \%)$ and normal systolic function (100.0\%) (LVEF $68.3 \% \pm 6.9 \%$, LVFS $37.3 \% \pm 5.9 \%)$. There were 3 children with small to moderate mitral regurgitation (42.9\%), 1 with light 
Table 2 Changes of myocardial enzyme at different time in 7 children with fulminant myocarditis implanted endocardial pacing

\begin{tabular}{lccccc}
\hline Myocardial enzyme & $1^{\text {st }}$ day $(\mathrm{n}=7)$ & $3^{\text {rd }}$ day $(\mathrm{n}=7)$ & $5^{\text {th }}$ day $(\mathrm{n}=7)$ & $7^{\text {th }}$ day $(\mathrm{n}=7)$ & $\mathrm{P}$ value \\
\hline CK $(\mathrm{U} / \mathrm{L})$ & $728.0(337.0,1465.0)$ & $75.0(34.0,109.0)$ & $34.0(26.0,55.0)$ & $24.0(21.0,34.0)$ & 0.000 \\
CKMB $(\mathrm{U} / \mathrm{L})$ & $62.0(12.0,97.0)$ & $6.0(3.0,21.0)$ & $4.0(3.0,10.0)$ & $3.0(2.0,4.0)$ & 0.006 \\
CKMBmass $(\mathrm{ng} / \mathrm{mL})$ & $38.8(24.8,75.3)$ & $2.7(1.4,11.5)$ & $2.1(0.8,3.1)$ & $1.2(1.1,1.8)$ & 0.001 \\
TnT $(\mathrm{ng} / \mathrm{mL})$ & $2.07(1.34,4.82)$ & $0.24(0.02,0.62)$ & $0.03(0.01,0.08)$ & $0.01(0.01,0.04)$ & 0.000 \\
\hline
\end{tabular}

$\mathrm{CK}$, creatine kinase; CKMB, creatine kinase-MB; TnT, troponin T.

pericardial effusion $(14.2 \%)$, and 3 left thickening of interventricular septum and left ventricular posterior wall (42.9\%). There were 2 children with endocardial pacing who did not revert to normal sinus rhythm, among whom1 had ventricular septal defect and atrial septal defect repair 7 years ago. Pacing rhythm persisted 7 days after the implantation of a temporary pacemaker and a permanent pacemaker was implanted after 1 week of hospitalization. The other was a child with fulminant myocarditis complicated with multiple malignant arrhythmias who developed liver failure, renal failure, and severe internal environment disorder. Her family gave up treatment 5 days after hospitalization. A total of 4 children with epicardial pacing were discharged from hospital 8-13 days after operation. The UCG showed that the diameter of heart chamber was significantly smaller than that before operation. The ECG showed normal sinus rhythm in 3 children and II atrioventricular block (II AVB) left in 1 child.

\section{Follow-up}

A total of12 children were followed up for $20.0 \pm 12.5$ months and 1 was lost follow-up. There were 7 children with endocardial pacing who had their temporary pacemakers

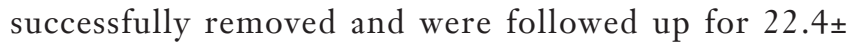
12.2 months. The ECG showed that normal sinus rhythm was restored in 5 children and complete right bundle branch block remained in 2 at 3 months after discharge. The UCG showed slight thickening of the interventricular septum in 2 children and normal diameter of heart chamber and cardiac function in the other 5 children. The ECG of the 1 child who was implanted with a permanent pacemaker revealed pacing rhythm and his UCG showed that the diameter of heart chamber was smaller than before and the systolic function was normal. He was treated with oral captopril only. We followed up 4 children with epicardial pacing for $6.0-18.0$ months. There were 3 children with no atrioventricular block and 1 child showed II AVB which had no progressive deterioration. The UCG showed that the diameter of heart chamber was normal and cardiac function was normal.

\section{Discussion}

A temporary pacemaker is an electronic device that stimulates the heart with electrical pulses to maintain or restore a normal heartbeat and recover circulatory function with non-permanent implantation of a pacing lead (1). The pacing mode in this study was VVI mode, which paced and sensed the ventricle and was suppressed by a sensed ventricular event (2).

In this study, fulminant myocarditis was the most common disease in children with temporary pacemakers. Among all cases of acute myocarditis, about $10-38 \%$ of cases involve fulminant myocarditis (13), which can be clinically differentiated from acute myocarditis by the presence of severe hemodynamic deterioration, cardiogenic shock, severe heart failure, and/or refractory life-threatening arrhythmias requiring inotropic support or mechanical cardiopulmonary assist devices (14). Fulminant myocarditis has higher rates of cardiac death and heart transplantation both in the short and long term (15). Early supporting of cardiac pump function and urgent management of serious arrhythmias has important clinical significance (16). In this study, all 8 children with fulminant myocarditis had CAVB and heart failure, which were in accordance with class I pacemaker implantation indication (17). By adjusting pacing parameters, the implantation of temporary pacemaker can provide effective pacing rate, increase cardiac output, improve hemodynamics, guarantee end-organ perfusion, and reduce organ injury. At the same time, it can increase coronary blood flow and improve myocardial blood supply, which is beneficial to the recovery of myocardial lesion. The implantation of a temporary pacemaker can stabilize heartbeat and improve myocardial function, so that it 
can correct pump failure and electrical failure caused by fulminant myocarditis. A total of 8 children with fulminant myocarditis were included in our study. The time from onset to admission of 7 children was $3 \mathrm{~h}$ to 2 days (mean $0.96 \pm$ 0.82 days) and the time from admission to implantation of temporary pacemakers was $2.7 \pm 1.1 \mathrm{~h}$. Combined with other drugs, the implantation of temporary pacemakers relieved clinical symptoms, stabilized hemodynamics gradually, improved cardiac function gradually, and decreased the level of myocardial enzyme gradually. The normal sinus rhythm was regained within $22.4 \pm 19.1 \mathrm{~h}$ and the working time of temporary pacemakers was $165.1 \pm 32.7 \mathrm{~h}$. All these 7 children had their temporary pacemakers removed successfully. The remaining child was on the $7^{\text {th }}$ day of the disease course at the time of admission, and she did not revert to normal sinus rhythm after 5 days of implantation of temporary pacemaker. Her family gave up further treatment because of severe myocardial lesion, multiple malignant arrhythmias, multiple organ failure, and severe internal environment disorder. The data showed that as a safe and effective first aid treatment, the timely implantation of a temporary pacemaker played an important role in successful rescue and improving prognosis in the treatment of fulminant myocarditis.

Severe bradycardia can contribute to a pronounced decrease in cardiac output followed by inadequate tissue perfusion and Adams-Stokes attacks which can aggravate heart damage resulting in a vicious circle. Most of the symptomatic bradycardia leading to the implantation of temporary pacemaker in children are CAVB. Except for myocarditis, the involvement of cardiac conduction system by various causes can lead to CAVB, some of which cannot be determined. Most of the CAVB with no known cause are not reversible, with most of these children requiring implantation of permanent pacemakers (18). One child with temporary endocardial pacemaker in this study had ventricular septal defect and atrial septal defect repair 7 years ago. More than 1 year before this admission, his parents found that his heart rate was occasionally slow but no further consultation was pursued. This time, the disease began with Adams-Stokes attack after exercise and the ECG showed CAVB. Fulminant myocarditis was considered at the beginning of admission. However, combined with blood examination and auxiliary examination after admission, the presentation was not in line with the criteria for the clinical diagnosis of myocarditis in the Diagnostic Recommendations for Children with Myocarditis (2018 edition) published by the Subspecialty Group of Cardiology of the Society of Pediatrics of Chinese Medical Association (19), and the diagnosis was revised to arrhythmia-CAVB. Pacing rhythm persisted 7 days after the implantation of temporary pacemaker which was in accordance with class I permanent pacemaker implantation indication (20). Finally, the permanent pacemaker was implanted. In this case, a temporary pacemaker was used as a bridge to permanent pacemaker when permanent pacing was not immediately indicated providing effective pacing rate, re-establishing normal hemodynamics, and avoiding organ failure and ventricular arrest caused by bradycardia (21).

In this study, on admission 1 child had prolonged QT interval and multiple TdP followed by Adams-Stokes attack and was defibrillated several times. A temporary pacemaker was implanted and programmed at a heart rate to terminate incessant TdP by decreasing QT intervals. Meanwhile, it was reported that defibrillation and/or overdriving by temporary pacemaker were helpful for termination of sustained ventricular tachycardia and $2.3 \%$ of temporary pacemakers were used to treat ventricular premature beats and ventricular tachycardia $(21,22)$.

Postoperative arrhythmias are common and occur in $7.5-48 \%$ of postoperative pediatric cardiac patients because of electrolytes disturbances, acidosis, hypotension, hypoxia, high catecholamine level, and local tissue edema and inflammation in the myocardium adjacent to conduction system. Many clinically significant arrhythmias can lead to hemodynamic deterioration, resulting in increased risk for mortality and morbidity $(8,9)$. In 2019, Jain et al. reported that the prevalence of arrhythmia (within 3 days of surgery) was $14.4 \%$ and complete heart block was the most common arrhythmia $(5.2 \%)$ in their study containing 536 pediatric cardiac surgical children (9). A temporary epicardial pacing wire is convenient and safe to be implanted in open heart surgery and a temporary pacemaker is important in the management of postoperative arrhythmia. Bradyarrhythmias, caused by either sinus node dysfunction or heart block, can be treated by a temporary pacemaker. At the same time, a temporary pacemaker is also a valuable diagnostic and therapeutic tool for children experiencing tachyarrhythmias such as junctional ectopic tachycardia and supraventricular tachyarrhythmia (8-10,23). Postoperative arrhythmia may recover over time and a temporary pacemaker can play a transitional role (24-26). In this study, 4 children with epicardial pacing developed CAVB after open heart surgery, which was in accordance with the pacemaker installation indication $(17,20)$. These 
4 children reverted to sinus rhythm within $1.5-5.0 \mathrm{~h}$ and had their temporary pacemakers successfully removed in 12.0-17.0 h. The temporary pacemaker played a critical role in maintaining circulatory stability in the early stage after open heart operation by overriding the intrinsic rate of the children, increasing cardiac output, and improving postoperative hemodynamics (26). At discharge, 3 children had no AVB and 1 left with II AVB. During the follow-up period (range, 6.0 to 18.0 months), there was no progressive deterioration in ECG and cardiac function was normal. These findings suggested that temporary pacemaker was convenient, effective, and could improve the prognosis in pediatric open heart surgery.

Although the general application for temporary pacemaker implantation in children are similar to those in adults, there are several important considerations in pediatric patients. First, the clinical significance of bradycardia is age dependent, so that the indications for pacemaker implantation need to be based on the correlation of symptoms with relative bradycardia rather than absolute heart rate criteria. Second, small physique, low tolerance to disease and cardiac anomaly make temporary pacemaker implantation more complex and sometimes face significant technical challenges. Third, there are no randomized clinical trials of cardiac pacing in pediatric or congenital heart disease patients (20). Due to the anatomical and physiological characteristics of children, selection of appropriate pacing mode, pacing site, and implantation route is very important (11). In a retrospective, observational analysis over a 10-year period, Czosek et al. reported that the total complication rate of pacemakers was $17.3 \%$, of which device-related was $7.2 \%$ and patientsrelated was $11.2 \%$ (27). In this study, VVI mode, single chamber, right ventricular apical pacing, and right femoral vein access were used in all children with temporary endocardial pacing. After operation, poor pacing occurred in 2 children and ECG showed intermittent pacing. After increasing the output voltage, the pacemaker worked well which was considered to be related to local tissue edema around the catheter. Catheter displacement occurred in 1 child due to his restlessness. The ECG showed that pacing signal disappeared and CAVB presented, followed by another Adams-Stokes attack. After re-adjusting the position of the pacing catheter, the pacemaker worked well which suggested that we should fix the catheter firmly and ensure proper tension during implantation and promptly calm the child down after operation. There were no related complications such as myocardial perforation, cardiac tamponade, pacemaker-related arrhythmia, catheter rupture, thromboembolism, or puncture site infection in all 9 children with temporary endocardial pacing. Among the complications associated with temporary epicardial pacing, the most concerning issue related to hemorrhage and late tamponade due to removal of the temporary pacemaker wires which may call for re-exploration and be lifethreatening $(28,29)$. None of the children with temporary epicardial pacing had related complications such as difficulty in wire removal or hemopericardium.

In this study, all children were equipped with bedside ECG monitoring and defibrillator. Experienced medical staff dynamically observed the cardiac electrical activity of children for 24 h-a-day. On the one hand, we should guard against the stimulation of pacing wires to trigger sympathetic storm under the condition of unstable myocardial electrical activity; on the other hand, combined with the changes of ECG, we should adjust pacing frequency, perceptual voltage, and output voltage when necessary. At present, the indication for temporary pacemaker removal has yet to achieve consensus. The working time of temporary pacemakers in this study was $134.0 \mathrm{~h}$ (15.0-191.0 h). In 7 children with endocardial pacing, temporary pacemakers were removed at $165.1 \pm$ $32.7 \mathrm{~h}$ when the condition was stable for more than $24 \mathrm{~h}$. In 4 children with epicardial pacing, temporary pacemakers were removed at 12.0-17.0 $\mathrm{h}$ when the hemodynamics were stable. Mastering the appropriate time to remove the pacemaker reduced the risk of complications and avoided the possible reduction of cardiac output due to the loss of the normal depolarization sequence of the heart in VVI pacing mode $(30,31)$.

Timely and correct treatment is the key factor determining curative effect and prognosis in the treatment of acute and critical cardiovascular diseases in children. As a technique with great clinical value, temporary pacemaker uses electrical impulse to stimulate heartbeat and stabilize hemodynamics. Our study demonstrated that the temporary pacemaker had the advantage of simple operation, definite effect, and safety, which had a remarkable effect in the treatment of acute and critical cardiovascular diseases in children.

This study has some limitations. This is a retrospective analysis of a single-center study using a small sample size, which may cause some deviations in the results. A large-sample multicenter study can further promote the application of temporary pacemakers in the treatment of acute and critical cardiovascular diseases in children. 


\section{Acknowledgments}

Funding: None.

\section{Footnote}

Reporting Checklist: The authors have completed the STROBE reporting checklist. Available at https:// tp.amegroups.com/article/view/10.21037/tp-21-586/rc

Data Sharing Statement: Available at https://tp.amegroups. com/article/view/10.21037/tp-21-586/dss

Conflicts of Interest: Both authors have completed the ICMJE uniform disclosure form (available at https://tp.amegroups. com/article/view/10.21037/tp-21-586/coif). The authors have no conflicts of interest to declare.

Ethical Statement: The authors are accountable for all aspects of the work in ensuring that questions related to the accuracy or integrity of any part of the work are appropriately investigated and resolved. All procedures performed in this study involving human participants were in accordance with the Declaration of Helsinki (as revised in 2013). The study was approved by Ethics and Research Committee of Tianjin Children's Hospital (No. L202118). Individual consent for this retrospective analysis was waived.

Open Access Statement: This is an Open Access article distributed in accordance with the Creative Commons Attribution-NonCommercial-NoDerivs 4.0 International License (CC BY-NC-ND 4.0), which permits the noncommercial replication and distribution of the article with the strict proviso that no changes or edits are made and the original work is properly cited (including links to both the formal publication through the relevant DOI and the license). See: https://creativecommons.org/licenses/by-nc-nd/4.0/.

\section{References}

1. Dalia T, Amr BS. Pacemaker Indications. 2021 Aug 30. In: StatPearls [Internet]. Treasure Island (FL): StatPearls Publishing, 2021.

2. Puette JA, Malek R, Ellison MB. Pacemaker. 2021 Sep 18. In: StatPearls [Internet]. Treasure Island (FL): StatPearls Publishing, 2021.

3. Writing Committee Members; Shah MJ, Silka MJ, et al. 2021 PACES Expert Consensus Statement on the Indications and Management of Cardiovascular Implantable Electronic Devices in Pediatric Patients. Heart Rhythm 2021;18:1888-924.

4. Kim J, Cho MJ. Acute Myocarditis in Children: a 10year Nationwide Study (2007-2016) based on the Health Insurance Review and Assessment Service Database in Korea. Korean Circ J 2020;50:1013-22.

5. Lv J, Han B, Wang C, et al. The Clinical Features of Children With Acute Fulminant Myocarditis and the Diagnostic and Follow-Up Value of Cardiovascular Magnetic Resonance. Front Pediatr 2019;7:388.

6. Fuchigami T, Nishioka M, Akashige T, et al. Pacemaker therapy in low-birth-weight infants. J Card Surg 2018;33:118-21.

7. Li XM, Zhang DY, Li HY, et al. Emergency Pacing via the Umbilical Vein and Subsequent Permanent Pacemaker Implantation in a Neonate. Pediatr Cardiol 2017;38:199-201.

8. Kabbani MS, Al Taweel H, Kabbani N, et al. Critical arrhythmia in postoperative cardiac children: Recognition and management. Avicenna J Med 2017;7:88-95.

9. Jain A, Alam S, Viralam SK, et al. Incidence, Risk Factors, and Outcome of Cardiac Arrhythmia Postcardiac Surgery in Children. Heart Views 2019;20:47-52.

10. Bar-Cohen Y, Silka MJ. Management of postoperative arrhythmias in pediatric patients. Curr Treat Options Cardiovasc Med 2012;14:443-54.

11. Takeuchi D, Tomizawa Y. Pacing device therapy in infants and children: a review. J Artif Organs 2013;16:23-33.

12. McLeod KA. Cardiac pacing in infants and children. Heart 2010;96:1502-8.

13. Devkota K, Wang YH, Liu MY, et al. Case Report: $\mathrm{III}^{\circ}$ atrioventricular block due to fulminant myocarditis managed with non-invasive transcutaneous pacing. F1000Res 2018;7:239.

14. Saji T, Matsuura H, Hasegawa K, et al. Comparison of the clinical presentation, treatment, and outcome of fulminant and acute myocarditis in children. Circ J 2012;76:1222-8.

15. Ammirati E, Veronese G, Brambatti M, et al. Fulminant Versus Acute Nonfulminant Myocarditis in Patients With Left Ventricular Systolic Dysfunction. J Am Coll Cardiol 2019;74:299-311.

16. Sharma AN, Stultz JR, Bellamkonda N, et al. Fulminant Myocarditis: Epidemiology, Pathogenesis, Diagnosis, and Management. Am J Cardiol 2019;124:1954-60.

17. Li XM. Pacing in atrioventricular conduction block. Chinese Pediatric Emergency Medicine 2017;24:649-52. 
18. Irfan M, Khan I, Ullah Bacha K. Delays in Temporary and Permanent Pacemakers: Causes and In-Hospital Outcomes. Cureus 2020;12:e6953.

19. Subspecialty Group of Cardiology, the Society of Pediatrics, Chinese Medical Association, et al. Diagnostic recommendation for myocarditis in children (version 2018). Chinese Journal of Pediatrics 2019;57:87-9.

20. Epstein AE, Dimarco JP, Ellenbogen KA, et al. ACC/AHA/ HRS 2008 Guidelines for device-based therapy of cardiac rhythm abnormalities. Heart Rhythm 2008;5:e1-62.

21. Tjong FVY, de Ruijter UW, Beurskens NEG, et al. A comprehensive scoping review on transvenous temporary pacing therapy. Neth Heart J 2019;27:462-73.

22. Yang D, Dai Q, Wu H, et al. The diagnostic capability of electrocardiography on the cardiogenic shock in the patients with acute myocarditis. BMC Cardiovasc Disord 2020;20:502.

23. Entenmann A, Dittrich S, Hessling V, et al. Standardized training for AVT pacing in paediatric patients with postoperative junctional ectopic tachycardia. Nurs Crit Care 2018;23:192-7.

24. The'Task Force on cardiac pacing and resynchronization therapy of the European Society of Cardiology (ESC). Developed in collaboration with the European Heart Rhythm Association (EHRA); Brignole M, Auricchio A, et al. 2013 ESC Guidelines on cardiac pacing and cardiac resynchronization therapy. Rev Esp Cardiol (Engl Ed) 2014;67:58.

Cite this article as: $\mathrm{Li}$ TT, Cheng $\mathrm{J}$. Clinical analysis of temporary pacemaker implantation in 13 children. Transl Pediatr 2022;11(2):174-182. doi: 10.21037/tp-21-586
25. Schernthaner C, Kraus J, Danmayr F, et al. Short-term pacemaker dependency after transcatheter aortic valve implantation. Wien Klin Wochenschr 2016;128:198-203.

26. Sullivan BL, Bartels K, Hamilton N. Insertion and Management of Temporary Pacemakers. Semin Cardiothorac Vasc Anesth 2016;20:52-62.

27. Czosek RJ, Meganathan K, Anderson JB, et al. Cardiac rhythm devices in the pediatric population: utilization and complications. Heart Rhythm 2012;9:199-208.

28. Bougioukas I, Jebran AF, Grossmann M, et al. Is there a correlation between late re-exploration after cardiac surgery and removal of epicardial pacemaker wires? J Cardiothorac Surg 2017;12:3.

29. Elmistekawy E, Gee YY, Une D, et al. Clinical and mechanical factors associated with the removal of temporary epicardial pacemaker wires after cardiac surgery. J Cardiothorac Surg 2016;11:8.

30. Reade MC. Temporary epicardial pacing after cardiac surgery: a practical review: part 1: general considerations in the management of epicardial pacing. Anaesthesia 2007;62:264-71.

31. Holmqvist F, Rathakrishnan B, Jackson LR 2nd, et al. Pacemaker programming in patients with first-degree AVblock: Programming pattern and possible consequences. Health Sci Rep 2018;1:e39.

(English Language Editor: J. Jones) 